\title{
On elliptic partial differential equations in bioimpedance
}

\author{
Ariungerel Jargal ${ }^{1} \cdot$ Jin Keun Seo ${ }^{1} \cdot$ Hyeuknam Kwon ${ }^{2}$ \\ Received: 15 February 2020 / Accepted: 13 April 2020 / Published online: 2 May 2020 \\ () The Author(s) 2020
}

\begin{abstract}
This paper deals with mathematical models in electrical bioimpedance fields that are described by means of elliptic partial differential equations (PDEs). To find solutions that have practical significance and value, it is necessary to gain a deep understanding of the underlying physical phenomena with the parameter details of PDE models as well as the data acquisition systems. This paper describes practical details of boundary conditions and effective coefficients of elliptic PDEs occurring in bioimpedance.
\end{abstract}

Keywords Elliptic partial differential equation · Maxwell's equations · Homogenization · Electrical bioimpedance

Mathematics Subject Classification $35 \mathrm{~A} 99 \cdot 35 \mathrm{~J} 05 \cdot 35 \mathrm{R} 30 \cdot 65 \mathrm{~N} 21$

\section{Introduction}

Elliptic partial differential equations (PDEs) are used to describe a large variety of physical phenomena. This paper focuses on PDE models in electrical bioimpedance area, which can be derived from suitable arrangements of Maxwell's equations at frequency below $1 \mathrm{MHz}$. Bioelectrical impedance methods are low cost, noninvasive and portable techniques for real-time clinical status monitoring (e.g. cardiopulmonary monitoring for patients with sleep apnea/obesity hypoventilation syndrome) $[29,57]$, estimating body composition (e.g. body fat and muscle mass) $[25,36,37]$,

Hyeuknam Kwon

hyeuknamkwon@yonsei.ac.kr

Ariungerel Jargal

j.ariungerel@gmail.com

Jin Keun Seo

seoj@yonsei.ac.kr

1 Department of Computational Science and Engineering, Yonsei University, Seoul, Korea

2 Department of Mathematics, Yonsei University, Wonju, Korea 
and others. Hence, it has been one of important research topics during the past four decades.

Bioelectrical impedance imaging is based on Ohm's law $\mathbf{J}=A \mathbf{E}$, where $A=\left(a_{i j}\right)$ is $3 \times 3$ matrix representing an electrical admittivity tensor, $\mathbf{E}$ is the electric field, and $\mathbf{J}$ is the current density. To generate $\mathbf{J}$ and $\mathbf{E}$ into a biological object occupying a three dimensional domain $\Omega \subset \mathbb{R}^{3}$ (with its diameter of less than 2 $\mathrm{m})$, we inject an electrical current by attaching several surface electrodes on the boundary $\partial \Omega$. Since $\nabla \times \mathbf{E} \approx 0$ at low frequency (below $1 \mathrm{MHz}$ ), the resulting electric field is $\mathbf{E}=-\nabla u$ where $u \in H^{1}(\Omega)$ is the electrical potential governed by the following elliptic PDE:

$$
\begin{cases}\sum_{i, j=1}^{3} \frac{\partial}{\partial x_{i}}\left(a_{i j} \frac{\partial}{\partial x_{j}} u\right)=0 & \text { in } \Omega, \\ \sum_{i, j=1}^{3} n_{i}\left(a_{i j} \frac{\partial}{\partial x_{j}} u\right)=g & \text { on } \partial \Omega,\end{cases}
$$

where $\mathbf{x}=\left(x_{1}, x_{2}, x_{3}\right)$ denotes position vector, $\mathbf{n}=\left(n_{1}, n_{2}, n_{3}\right)$ is the unit outer normal vector to the boundary $\partial \Omega$, and $g \in H^{-1 / 2}(\partial \Omega)$ represents Neumann boundary data corresponding to the injected current. Note that $g$ must satisfy $\int_{\partial \Omega} g d s=0$ for the existence and $u$ is usually normalized by $\int_{\partial \Omega} u d s=0$ for the uniqueness, where $d s$ denotes the surface element.

The coefficient $A$ should be understood as a homogenized admittivity tensor that depends on scale, position, cell structure including molecular compositions of cells, shape and direction of cells, cellular membranes, intra-and extra-cellular fluids, concentrations and mobilities of ions [60]. For the existence and uniqueness of a weak solution according to Lax-Milgram theorem [11], the coefficient $A$ in $\Omega$ must satisfy

$$
0<\lambda_{\min }|\xi|^{2} \leq \sum_{i, j=1}^{3} a_{i j}(\mathbf{x}) \xi_{i} \xi_{j} \leq \lambda_{\max }|\xi|^{2} \quad \text { for all } \mathbf{x} \in \Omega, \xi \in \mathbb{R}^{3} \backslash\{0\}
$$

where $\lambda_{\min }$ and $\lambda_{\max }$ are positive constants. For stability, the ratio $\lambda_{\max } / \lambda_{\min }$ should not be too large. The admittivity tensor $A$ can be derived by asymptotic homogenization techniques with knowledge of pointwise admittivity distribution [3, 18, 19, 44]. However, the pointwise admittivity may not exist in real world, because the admittivity is passive property [9]. What we can observe is its effective or equivalent property $[14,43]$. The coefficient $A$ in muscle region usually exhibits a strong anisotropy depending on muscle fiber orientation and scale [10]. The coefficient $A$ of tissue is affected by functional and pathological conditions such as ischemia, hemorrhage, edema, inflammation, cancers, and neural activities [60]. Hence, the problem of estimating $A$ of tissue has been an important research topic in biomedical field $[25,29,57,58]$.

In practice, we cannot know the Neumann data $g$ in (1) accurately. The $g$ is determined by the configuration of the array of surface electrodes (that are attached on $\partial \Omega$ ) and the current injection pattern. If we use a pair of surface 
electrodes to inject a dc current of $I \mathrm{~mA}$ into the subject $\Omega$, the induced electrical field $-\nabla u$ inside $\Omega$ roughly satisfies the following boundary conditions:

$$
\left\{\begin{aligned}
(A \nabla u) \cdot \mathbf{n} & =0 \quad \text { on } \partial \Omega \backslash\left(\mathcal{E}_{+} \cup \mathcal{E}_{-}\right) \\
\int_{\mathcal{E}_{+}} A \nabla u \cdot \mathbf{n} d s & =I=-\int_{\mathcal{E}_{-}} A \nabla u \cdot \mathbf{n} d s \\
\mathbf{n} \times \nabla u & =0 \quad \text { on } \mathcal{E}_{+} \cup \mathcal{E}_{-}
\end{aligned}\right.
$$

where $\mathcal{E}_{+}$and $\mathcal{E}_{-}$denote the skin-electrode contact area as shown in Fig. 1. Here, the skin-electrode contact impedance [61] is ignored for mathematical simplicity. Note that the corresponding Neumann data $g=\nabla u \cdot \mathbf{n}$ is living in the Sobolev space $H^{-1 / 2}(\partial \Omega)$ and $g$ is unbounded due to its singularity on the edge of $\mathcal{E}_{ \pm}$. This boundary condition will be discussed in detail.

We need to choose the domain $\Omega$ such that the boundary value problem (1) is well-posed in the sense of Hadamard [26]. Consequently, it is necessary to exclude regions with almost extreme (zero or infinite) admittivity from $\Omega$. For example, the air region in stomach has almost zero admittivity and electrodes has almost infinite admittivity. We should note that the Neumann data $g$ is very limited depending on the placement and number of electrodes. In order to apply rapidly oscillating boundary data $g$, we need to attach many electrodes, resulting in the electrode-skin contact area occupying a large portion of $\partial \Omega$. In this case, unfortunately, most of electrical current flows significantly through the highly conducting electrodes. Hence, in the case when the electrode-skin contact area occupying a large portion of $\partial \Omega$, it would be desirable to include electrode regions to $\Omega$ for accurate analysis. There was often a misunderstanding of this issue with respect to $\Omega$ and $g$ in the both mathematics and engineering community. This paper describes this issue in detail.

Understanding the practical limitations and fundamental physical phenomena mentioned above is important for solving various problems in bioimpedance including electrical impedance tomography (EIT) and electric impedance myography(EIM). EIT and EIM are to estimate the electrical tissue property $A$ in (1) of biological subject at various frequency below $1 \mathrm{MHz}$. With some ideal assumptions, the mathematical formulation of EIT system can be described as the well known Calderón problem which aims to identify an equivalent isotropic conductivity $A$ from the Neuman-to-Dirichlet map $\mathfrak{\Omega}_{A}:\left.g \in H^{-1 / 2}(\partial \Omega) \rightarrow u\right|_{\partial \Omega} \in H^{1 / 2}(\partial \Omega)$, where $u$ is the solution of the boundary
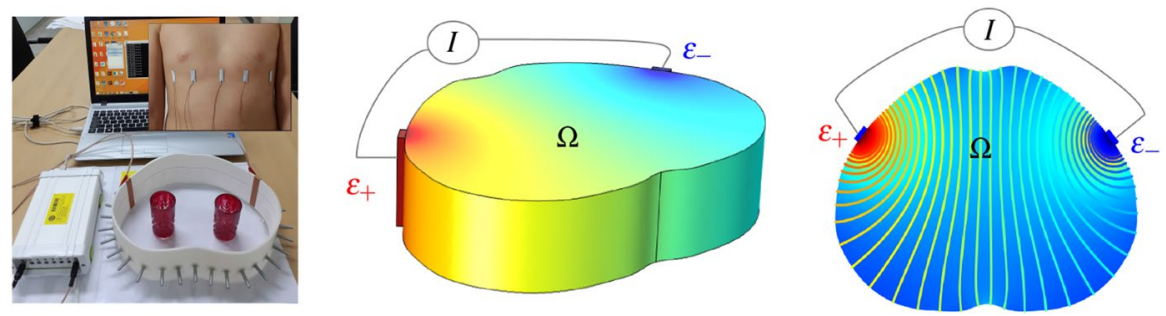

Fig. 1 Boundary value problem in (1). The Neumann boundary data is determined by the injection current applied using the pair of electrodes $\mathcal{E}_{+}$and $\mathcal{E}_{-}$ 
value problem (1). There have been obtained novel theoretical results guaranteeing a unique identification of isotropic $A$ from the NtD data $[4,8,33,35,45,46$, 62]. The analysis of PDE models often plays an important role in the achievement of major advances in these areas. However, it should not be studied in isolation, and practical limitations associated with the measurement sensitivity and specificity, noise, interface between $\Omega$ and instrument and so on must be properly understood and analyzed [57].

This paper is organized as following. Section 2 is devoted to explain elliptic PDE as a governing equation in bioimpedance and effective coefficient $A$ of the PDE. In Sect. 3, we introduce inverse problems of finding coefficient of the PDE; electrical impedance myography and electrical impedance tomography. Finally, we conclude the manuscript with discussion in Sect. 4.

\section{Boundary value problem}

\subsection{Elliptic PDE in bioimpedance}

The admittivity distribution $A$ in an imaging domain $\Omega$ (a part of human body) is the passive electrical property which can be estimated by Ohm's law, using the relationship between the Neumann data $g$ and the Dirichlet data $\left.u\right|_{\partial \Omega}$ with $u$ being the solution of the elliptic PDE (1). This boundary value problem is derived from Maxwell's equations [57]. In order to measure $A$, we inject a sinusoidal current $I \sin (\omega t)$ between a pair of electrodes $\left(\mathcal{E}_{+}\right.$and $\left.\mathcal{E}_{-}\right)$to produce the current density $\Re\left(\mathbf{J} e^{i \omega t}\right)$ and the electric field $\Re\left(\mathbf{E} e^{i \omega t}\right)$ inside $\Omega$. Here, $i=\sqrt{-1}, \mathfrak{R}(f)$ is the real part of $f, \omega$ is angular frequency, $t$ is time, $\mathbf{E}$ is the time-harmonic electric field, and $\mathbf{J}$ is the timeharmonic current density. Throughout this paper, we assume that the boundary $\partial \Omega$ is smooth, electrodes $\mathcal{E}_{ \pm}$are rectangular shape as shown in Fig. 1, the diameter of $\Omega$ is less than $1 \mathrm{~m}$, and the applied frequency $\omega / 2 \pi$ is below $1 \mathrm{MHz}$.

For the given injection current, the corresponding Neumann data $g=\left.\mathbf{J} \cdot \mathbf{n}\right|_{\partial \Omega}$ satisfies

$$
\int_{\mathcal{E}_{+}} g d s=I=-\int_{\mathcal{E}_{-}} g d s, \quad g=0 \quad \text { on } \partial \Omega \backslash\left(\mathcal{E}_{+} \cup \mathcal{E}_{-}\right) .
$$

From the time-harmonic Maxwell's equation, we have

$$
\nabla \times \mathbf{E}=-i \omega \mu_{0} \mathbf{H}, \quad \nabla \times \mathbf{H}=\mathbf{J}, \quad \mathbf{J}=A \mathbf{E} \quad \text { in } \Omega
$$

where $\mathbf{H}$ is the time-harmonic magnetic field and $\mu_{0}=4 \pi \times 10^{-7} \mathrm{H} / \mathrm{m}$ is the magnetic permeability of free space. The human body is assumed to be the constant $\mu_{0}$. Assuming that $\omega \mu_{0} \mathbf{H} \approx 0$ at frequency below $1 \mathrm{MHz}$, there exists a complex potential $u$ such that $-\nabla u \approx \mathbf{E}$. Since $\nabla \cdot(\nabla \times \mathbf{H})=0$, it follows from (5) that $u$ satisfies the elliptic PDE (1) with $g$ being $\left.\mathbf{n} \cdot \mathbf{J}\right|_{\partial \Omega}$.

Note that $A$ can be expressed as $A=\sigma+i \omega \epsilon$, where $\sigma$ and $\epsilon$ are the conductivity and permittivity, respectively. The $A=A(\mathbf{x}, \omega)$ depends not only on the position 
$\mathbf{x}=\left(x_{1}, x_{2}, x_{3}\right)$ but also on the angular frequency $\omega$. The effective admittivity tensor $A$ (macroscopic scale) in $\Omega$ satisfies the usual ellipticity condition:

$$
c|\xi|^{2} \leq \Re(\langle A \xi, \xi\rangle) \leq c^{-1}|\xi|^{2}, \quad \xi \in \mathbb{C}^{3}
$$

where $c$ is a positive constant being away from zero. We should note that $A$ in a very microscopic scale may not satisfy the ellipticity condition (6) with $c \gtrsim 0$. It is because cell membranes are almost electrically insulating $(A \approx 0)$ at $\omega=0$.

For the ease of explanation, we focus on $A$ at dc current $(\omega=0)$. Let us understand the Neumann data $g$ and $\mathbf{J}=A \nabla u$ on the electrode area $\mathcal{E}_{+} \cup \mathcal{E}_{-}$. Since electrodes are highly conductive materials such as copper and carbon, we have $\mathbf{n} \times\left.\mathbf{J}\right|_{\mathcal{E}_{+} \cup \mathcal{E}_{-}} \approx 0$, under the assumption that the contact impedance underneath the electrode is ignored. Hence, the complex potential $u$ approximately satisfies

$$
\left\{\begin{array}{rlrl}
\nabla \cdot(A \nabla u) & =0 & & \text { in } \Omega \\
\int_{\mathcal{E}_{+}} A \nabla u \cdot \mathbf{n} d s & =I=-\int_{\mathcal{E}_{-}} A \nabla u \cdot \mathbf{n} d s \\
(A \nabla u) \cdot \mathbf{n} & =0 & & \text { on } \partial \Omega \backslash\left(\mathcal{E}_{+} \cup \mathcal{E}_{-}\right) \\
\mathbf{n} \times \nabla u & =0 & & \text { on } \mathcal{E}_{+} \cup \mathcal{E}_{-}
\end{array}\right.
$$

Setting $\int_{\partial \Omega} u d s=0$, we obtain a unique solution $u \in H^{1}(\Omega)$.

On the edge of $\mathcal{E}_{ \pm}$, there exists a singularity of $g$. The following observation explains the edge singularity on the electrodes.

Observation 2.1 Let $u$ be a solution of (7). Let $g=\left.\mathbf{n} \cdot \nabla u\right|_{\partial \Omega}$. Then, $g \in L^{p}(\partial \Omega)$ for $1 \leq p<2$ but $g \notin L^{2}(\partial \Omega)$. Moreover, $\|\nabla u\|_{L^{2}\left(\partial \Omega \backslash\left(\mathcal{E}_{+} \cup \mathcal{E}_{-}\right)\right)}=\infty$.

The proof is based on the square root edge singularity [23, 51] on the electrodes. For the ease of explanation, assume that $A$ is the identity matrix and $\Omega=\mathbb{R}_{-}^{3}=\left\{\mathbf{x}: x_{3}<0\right\}$ (the lower half space). We also assume that both $\mathcal{E}_{+}$and $\mathcal{E}_{-}$are rectangular domains lying on $\partial \Omega$. Let $w$ be the extension of $u$ such that $w\left(x_{1}, x_{2}, x_{3}\right)=w\left(x_{1}, x_{2},-x_{3}\right)$ (even reflection) for all $x_{3}>0$. Then, $w$ satisfies $\nabla \cdot \nabla w=0$ in $\mathbb{R}^{3} \backslash\left(\mathcal{E}_{+} \cup \mathcal{E}_{-}\right)$. Moreover, $\left.w\right|_{\mathcal{E}_{+}}$and $\left.w\right|_{\mathcal{E}_{-}}$are constants. Hence, $\nabla w$ has the square root edge singularity on $\mathcal{E}_{+} \cup \mathcal{E}_{-}$, resulting in

$$
\|g\|_{L^{2}\left(\mathcal{E}_{+} \cup \mathcal{E}_{-}\right)}=\|\nabla w\|_{L^{2}\left(\mathcal{E}_{+} \cup \mathcal{E}_{-}\right)}=\infty
$$

Now, it remains to prove $\|\nabla u\|_{L^{2}\left(\mathcal{E}_{+} \cup \mathcal{E}_{-}\right)}=\infty$. It can be proven the well-known Rellich type identity [50]. For any smooth vector field $F=\left(F_{1}, F_{2}, F_{3}\right)$, we have

$$
\nabla \cdot\left(F|\nabla u|^{2}\right)=(\nabla \cdot F)|\nabla u|^{2}+F \cdot \nabla(\nabla u \cdot \nabla u)
$$

Integrating the above identity over $\Omega$, we obtain

$$
\int_{\partial \Omega} F \cdot \mathbf{n}|\nabla u|^{2} d s=2 \int_{\partial \Omega}(F \cdot \nabla u)(\mathbf{n} \cdot \nabla u) d s+O\left(\|\nabla u\|_{L^{2}(\Omega)}^{2}\right)
$$

where $O\left(\|\nabla u\|_{L^{2}(\Omega)}^{2}\right)$ is used to express a term bounded by $\|\nabla u\|_{L^{2}(\Omega)}^{2}$. The identity (10) follows from 


$$
\begin{aligned}
F \cdot \nabla|\nabla u|^{2} & =\alpha \cdot \nabla\left(\sum_{j=1}^{3} \partial_{j} u \partial_{j} u\right) \\
& =2 F_{k}\left(\sum_{j=1}^{3} \partial_{k} \partial_{j} u \partial_{j} u\right) \\
& =2 \nabla \cdot((F \cdot \nabla u) \nabla u)-2 \sum_{j, k=1}^{3} \partial_{j} F_{k} \partial_{k} u \partial_{j} u
\end{aligned}
$$

Since $g=0$ in $\partial \Omega \backslash\left(\mathcal{E}_{+} \cup \mathcal{E}_{-}\right)$and $\mathbf{n} \times \nabla u=0$ on $\mathcal{E}_{+} \cup \mathcal{E}_{-}$, the identity (10) leads to

$$
\int_{\partial \Omega \backslash\left(\mathcal{E}_{+} \cup \mathcal{E}_{-}\right)} F \cdot \mathbf{n}|\nabla u|^{2} d s=\int_{\mathcal{E}_{+} \cup \mathcal{E}_{-}}(F \cdot \mathbf{n})|g|^{2} d s+O\left(\|\nabla u\|_{L^{2}(\Omega)}^{2}\right) .
$$

Since the smooth vector field $F$ is arbitrary, it follows from (11) that $\|g\|_{L^{2}\left(\mathcal{E}_{+} \cup \mathcal{E}_{-}\right)}=\infty$ implies $\|\nabla u\|_{L^{2}\left(\partial \Omega \backslash\left(\mathcal{E}_{+} \cup \mathcal{E}_{-}\right)\right)}=\infty$. This proves Observation 2.1 for the case where $A$ is the identity matrix and $\Omega=\left\{\mathbf{x}: x_{3}<0\right\}$ (the lower half space). The general case can be similarly proved by a proper modification.

Next, we explain how the potential $u$ changes as additional electrodes $\left(\mathcal{E}_{1}, \mathcal{E}_{2}, \cdots, \mathcal{E}_{n}\right)$ are attached between $\mathcal{E}_{+}$and $\mathcal{E}_{-}$. See Fig. 2. Let all electrodes be the same size and shape, and let the spacing between electrodes be a constant $s_{n}$. We denote

$$
Y_{n}=\mathcal{E}_{+} \cup \mathcal{E}_{-} \cup \mathcal{E}_{1} \cup \cdots \cup \mathcal{E}_{n}
$$

The following observation shows that the boundary value problem (7) changes as the additional electrodes $Y_{n}$ increase.
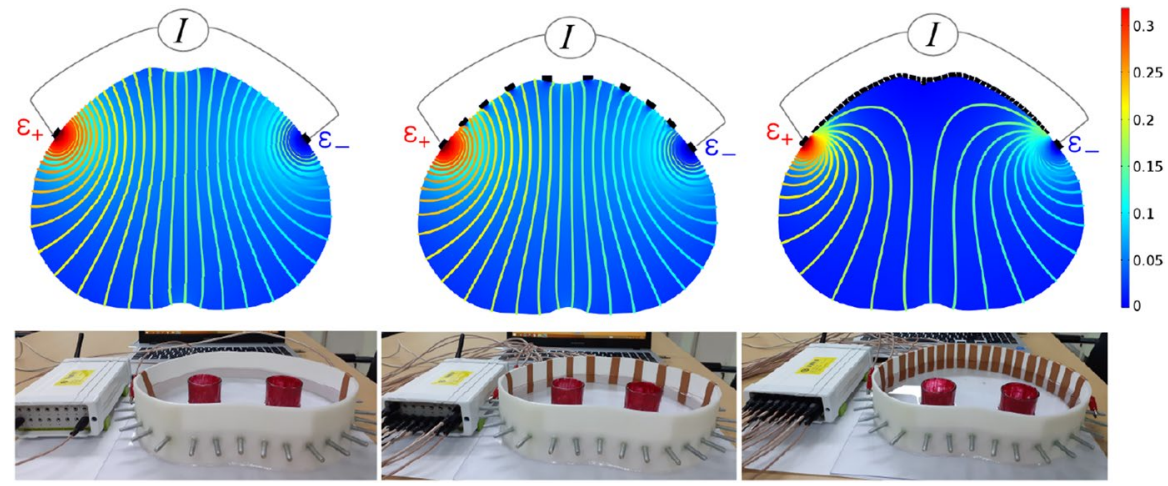

Fig. 2 Changes in Neumann boundary conditions even when the same current is applied through the same pair of electrodes $\left(\mathcal{E}_{+}\right.$and $\mathcal{E}_{-}$). The left image (as a solution of (7)) is almost the same as the middle image because $s_{n}$ (the spacing between electrodes described in Observation 2.2) is not so small. However, the right image is very different from the left image because $s_{n} \approx 0$ 
Observation 2.2 Let $u_{n}$ be the potential corresponding to $u$ in (7) with adding electrodes $\left(\mathcal{E}_{1}, \mathcal{E}_{2}, \cdots, \mathcal{E}_{n}\right)$ between $\mathcal{E}_{+}$and $\mathcal{E}_{-}$, as shown in Fig.2. The potential $u$ and $u_{n}$ satisfy the same first two conditions in (7) (because of the same $A, \Omega$, and the same injection current), but the last two boundary conditions are different. The last boundary conditions in (7) should be replaced by:

$$
\left\{\begin{aligned}
\left(A \nabla u_{n}\right) \cdot \mathbf{n}=0 & \text { on } \partial \Omega \backslash Y_{n} \\
\mathbf{n} \times \nabla u=0 & \text { on } Y_{n}
\end{aligned}\right.
$$

Moreover, as $s_{n} \rightarrow 0$ (the spacing between electrodes converges to zero), the potential difference $\left.u_{n}\right|_{\mathcal{E}_{+}}-\left.u_{n}\right|_{\mathcal{E}_{-}}$converges to zero and

$$
\left|\nabla u_{n}(\mathbf{x})\right| \rightarrow 0 \quad \text { for all } \mathbf{x} \in \Omega_{\delta}:=\{\mathbf{x} \in \Omega: \operatorname{dist}(\mathbf{x}, \partial \Omega)>\delta\}
$$

where $\delta$ is any positive constant and $\operatorname{dist}(\mathbf{x}, \partial \Omega)$ is the distance between $\mathbf{x}$ and $\partial \Omega$.

In electrical impedance tomography, several researchers have tried to use as many electrodes as possible in order to get a rough version of Neumann-to-Dirichlet map $[8,35]$. However, if the spacing between electrodes is very small due to many electrodes, it is almost impossible to get any information of $A$ in a remote internal region from the boundary, according to (14) in Observation 2.2.

For the well-posedness of the boundary value problem (1) from Lax-Milgram theorem, we usually exclude very high and low conducting region such as electrode and air to satisfy the condition (6). Indeed, in the case when a small number of electrodes are attached on $\partial \Omega$, solution $u$ of (1) can be approximated by that of (1) with excluding electrode region. However if electrodes almost surround the object then the approximation fails, because electric currents mostly flow boundary as shown in Fig. 2.

For the determination of $A$, according to the Calderón problem [8], it is necessary to have full boundary data which requires closely packed electrodes on the boundary. Most of practical studies (e.g. EIT, EIM) of finding $A$ in (1) from a partial information of Neumann-to-Dirichlet map $\mathfrak{Q}:\left.g \mapsto u\right|_{\partial \Omega}$ use less than or equal to 16 small electrodes.

\subsection{The effective coefficient $A$ of heterogeneous media and homogenization}

The effective admittivity tensor $A$ in a cubic voxel $Q_{\mathbf{x}}$ centered at a position $\mathbf{x}$ can be determined by Ohm's law:

$$
\int_{Q_{\mathbf{x}}} \mathbf{J}\left(\mathbf{x}^{\prime}\right) d \mathbf{x}^{\prime} \approx A(\mathbf{x}) \int_{Q_{\mathbf{x}}} \mathbf{E}\left(\mathbf{x}^{\prime}\right) d \mathbf{x}^{\prime}
$$

where $\mathbf{E}$ and $\mathbf{J}$ are the time-harmonic electric field and current density, respectively, in (5). Since the time-harmonic fields change with the angular frequency $\omega$, the effective admittivity tensor $A$ of biological tissue changes with $\omega$. Hence, $A=A(\mathbf{x}, \omega)$ depends on position $\mathbf{x}$, the applied frequency $\omega$, and the size of the voxel. The effective tensor $A$ is decomposed into its real and imaginary parts: 


$$
A(\mathbf{x}, \omega)=\underbrace{\sigma(\mathbf{x}, \omega)}_{\text {conductivity }}+i \omega \underbrace{\epsilon(\mathbf{x}, \omega)}_{\text {permittivity }}
$$

The effective coefficient $A$ can be derived from the pointwise admittivity, denoted by $A_{\mathrm{pt}}=\sigma_{\mathrm{pt}}+i \omega \epsilon_{\mathrm{pt}}$, on the basis of the two-scale homogenization theory [2]. The frequency dependency behavior of $A$ is owing to Maxwell-Wagner polarization effect [20], that is related to the geometry of biological tissue structure with cells, extracellular matrix, intra- and extra-cellular fluids [21,24]. The frequency-dependent $A$ is mainly influenced by the cell membranes with their thickness being in the order of several nm [34].

Let us understand intuitively how the effective conductivity $A$ is affected by the tissue structure. At low frequency, cell membranes are insulators and current can flow around them. As cell swelling occurs, the extra-cellular space within the region decreases and this results in a reduce effective conductivity. If cells are densely packed, the effective conductivity becomes smaller for the same reason. If cells are tightly packed along the horizontal direction and the gaps between cells in the vertical direction are bigger, then the effective conductivity will be different depending on the direction of the applied electric field. This means that we should consider the anisotropy as well as the inhomogeneity in the effective conductivity.

In 1924, Fricke [18, 19] described a mathematical form of the homogenized coefficient in the simplest case of dilute single suspension. Ammari et al [3] analyzed the role of the membrane in terms of the frequency-dependent behavior of a solution $u$ in the framework of the elliptic PDE in two dimensional case where cell membranes are immersed in a domain $\Omega$. For a rigorous analysis, they assume the followings: (1) $\Omega$ is divided periodically in each direction in identical very small squares and a cell lives in each small squares. (2) The medium outside the cell membranes is a homogeneous isotropic medium with $A_{\mathrm{pt}}^{0}=\sigma_{\mathrm{pt}}^{0}+i \omega \sigma_{\mathrm{pt}}^{0}$ and each thin membrane has the uniform thickness of $d \approx 0$ with the isotropic admittivity $A_{\mathrm{pt}}^{m}=\sigma_{\mathrm{pt}}^{m}+i \omega \sigma_{\mathrm{pt}}^{m}$ with $\frac{\sigma_{\mathrm{pt}}^{m}}{\sigma_{\mathrm{pt}}^{0}} \approx 0$. Fig. 3 illustrates the above assumptions more clearly, where the normalized square $[0,1]^{2}$ contains the reference cell membrane given by $\{\mathbf{x}$ : dist $(\mathbf{x}, \Gamma)<d\}$ for a cell contour $\Gamma$. Let us denote by $c$ the area of $\Gamma_{\text {inside }}$, which $\Gamma_{\text {inside }}$ is the domain inside the cell contour $\Gamma$, as shown in Fig. 3. We assume that $d / c \approx 0$. Given an applied sinusoidal current at the angular frequency $\omega$, the resulting harmonic potential $u$ in term of its pointwise coefficient $A_{\mathrm{pt}}$ approximately satisfies
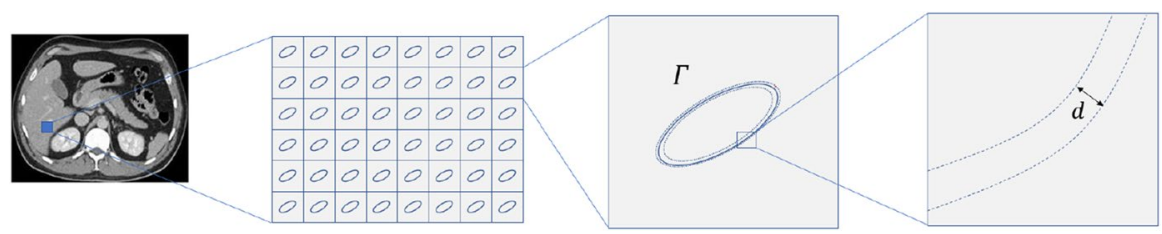

Fig. 3 Homogenization of $2 \mathrm{D}$ cell model with periodic structure. In this toy model, the membrane $\{\mathbf{x} \in Q: \operatorname{dist}(\mathbf{x}, \Gamma)<d\}$ is immersed in the cube $Q$, where $\Gamma$ is the cell contour 


$$
\begin{cases}\nabla \cdot \nabla u=0 & \text { in } \Omega \backslash \Gamma \\ {\left[\frac{\partial u}{\partial \mathbf{n}}\right] \approx 0} & \text { on } \Gamma \\ {[u] \approx \frac{d A_{\mathrm{pt}}^{0}}{A_{\mathrm{pt}}^{m}} \frac{\partial u}{\partial \mathbf{n}}} & \text { on } \Gamma\end{cases}
$$

where $[w(\mathbf{x})]$ represent the jump across the cell membrane $\Gamma$ : $[w(\mathbf{x})]=\left.w\right|_{+}-\left.w\right|_{-}=\lim _{s \rightarrow 0^{+}}[w(\mathbf{x}+s \mathbf{n})-w(\mathbf{x}-s \mathbf{n})]$ for $\mathbf{x} \in \Gamma$.

Ammari et al [3] obtained a formula for the effective $A$ of a periodic dilute suspension, under the above mentioned assumptions: For small $c$, the effective $A$ can be approximated by

$$
A \approx A_{\mathrm{pt}}^{0}\left(I+c M\left(I-\frac{c}{2} M\right)^{-1}\right)+o\left(c^{2}\right)
$$

where

$$
M=\left(-\frac{d A_{\mathrm{pt}}^{0}}{A_{\mathrm{pt}}^{m}} \int_{\Gamma / \sqrt{c}} n_{j}\left(I+\frac{d A_{\mathrm{pt}}^{0}}{A_{\mathrm{pt}}^{m}} \mathcal{L}_{\Gamma / \sqrt{c}}\right)^{-1}\left[n_{i}\right] d s\right)_{i, j \in\{1,2\}}
$$

and

$$
\mathcal{L}_{\Gamma / \sqrt{c}}[\phi](\mathbf{x})=\frac{1}{2 \pi} \int_{\Gamma / \sqrt{c}} \frac{\partial^{2} \ln \left|\mathbf{x}-\mathbf{x}^{\prime}\right|}{\partial \mathbf{n}(\mathbf{x}) \partial \mathbf{n}\left(\mathbf{x}^{\prime}\right)} \phi\left(\mathbf{x}^{\prime}\right) d s_{\mathbf{x}^{\prime}} .
$$

Here, $\Gamma / \sqrt{c}$ is the rescaled cell contour so that the operator $\mathcal{L}_{\Gamma / \sqrt{c}}$ is independent of $c$.

Although the concept of effective admittivity has been studied deeply using homogenization concepts, its practical and intuitive definition is not clear due to the ideal assumptions of periodic and dilute suspension. Seo et al [59] provided a practical way to measure the effective admittivity $A$ for a given cube from measurable boundary current-voltage data. Given a small cubic subject $Q \subset \Omega$, the effective admittivity $A$ can be computed by its pointwise admittivity $A_{\mathrm{pt}}$, as in homogenization approaches. Since $A(\omega)$ as a function of $\omega$ is regarded as an ensemble average of pointwise admittivity $A_{\mathrm{pt}}$ over the cube $Q$, the $A$ must satisfy

$$
\int_{Q} A_{\mathrm{pt}}(\mathbf{x}, \omega) \nabla u_{j} d \mathbf{x} \approx A(\omega) \int_{Q} \nabla u_{j} d \mathbf{x} \quad \text { for } j=1,2,3
$$

where $u_{j}$ is the solution of

$$
\left\{\begin{aligned}
\nabla \cdot\left(A_{\mathrm{pt}}(\mathbf{x}, \omega) \nabla u_{j}(\mathbf{x})\right) & =0 \quad \text { for } \mathbf{x} \in Q \\
\int_{\partial Q} \frac{\left|n_{j}\right|+n_{j}}{2} \mathbf{n} \cdot \nabla u_{j} d s & =I=\int_{\partial Q} \frac{-\left|n_{j}\right|+n_{j}}{2} \mathbf{n} \cdot \nabla u_{j} d s \\
\left(n_{j}\right) \mathbf{n} \times\left.\nabla u_{j}\right|_{\partial Q} & =0,\left.\left(1-\left|n_{j}\right|\right) \mathbf{n} \cdot \nabla u_{j}\right|_{\partial Q}=0
\end{aligned}\right.
$$




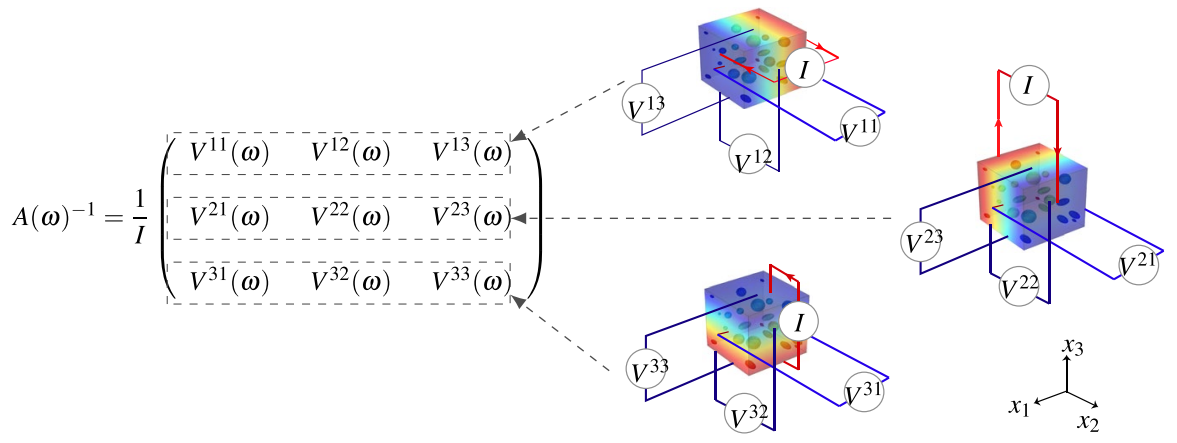

Fig. 4 Evaluation of the overall effective admittivity $A$ of a cubic heterogeneous subject
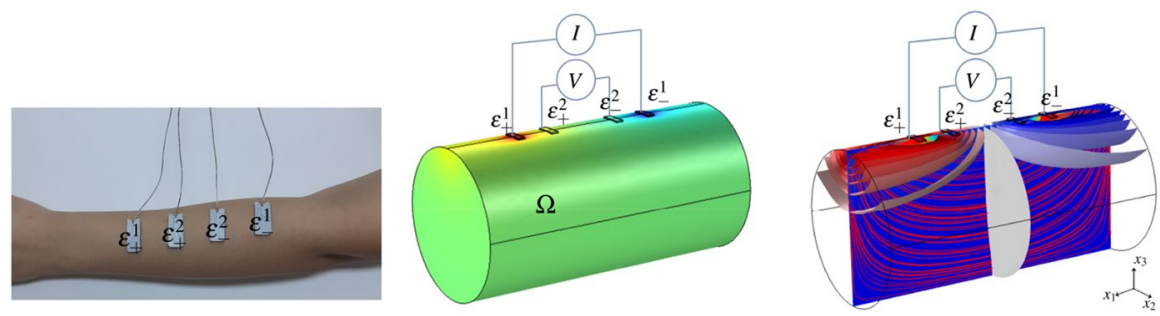

Fig. 5 Four electrode method for bioimpedance measurement. The pair of the outer electrodes $\left(\mathcal{E}_{+}^{1}\right.$ and $\left.\mathcal{E}_{-}^{1}\right)$ is used to inject current. The induced voltage is measured using the remaining pair $\left(\mathcal{E}_{+}^{2}\right.$ and $\left.\mathcal{E}_{-}^{2}\right)$

Here, we apply a sinusoidal current of $I \mathrm{~mA}$ at angular frequency $\omega$. For each pair $(j, k) \in\{1,2,3\}^{2}$, we denote the voltage difference between two sides of the cube $Q$ :

$$
V^{j k}(\omega)=\int_{\partial Q} n_{k} u^{j} d s \quad(j, k=1,2,3) .
$$

Then, the effective $A$ in the cube $Q$ can be evaluated as in Fig 4.

\section{Estimation of $A$ in electrical bioimpedance}

In bioimpedance, the simplest way of measuring an overall version of the effective admittivity $A$ of the biological subject (occupying a three dimensional domain $\Omega$ ) is the four-electrode method [24], as shown in Fig. 5; the pair of external electrodes (denoted by $\mathcal{E}_{+}^{1}$ and $\mathcal{E}_{-}^{1}$ ) is used to inject a sinusoidal current of $I \mathrm{~mA}$ at angular frequency $\omega$ and the induced voltage difference is measured the pair of inner electrodes (denoted by $\mathcal{E}_{+}^{2}$ and $\mathcal{E}_{-}^{2}$ ). et $u_{1}$ denote the induced potential due to the injection current using $\mathcal{E}_{+}^{1}$ and $\mathcal{E}_{-}^{1}$. Then $u_{1}$ is governed by 


$$
\left\{\begin{array}{rlr}
\nabla \cdot\left(A(\mathbf{x}, \omega) \nabla u_{1}(\mathbf{x})\right) & =0 \quad \text { for } \mathbf{x} \in \Omega \\
\int_{\mathcal{E}_{+}^{1}} \mathbf{n} \cdot\left(A \nabla u_{1}\right) d s & =I=-\int_{\mathcal{E}_{-}^{1}} \mathbf{n} \cdot\left(A \nabla u_{1}\right) d s \\
\int_{\mathcal{E}_{+}^{2}}^{2} \mathbf{n} \cdot\left(A \nabla u_{1}\right) d s & =0=\int_{\mathcal{E}_{-}^{2} \mathbf{n} \cdot\left(A \nabla u_{1}\right) d s} \\
\mathbf{n} \times \nabla u_{1} & =0 \quad \text { on } \mathcal{E}_{+}^{1} \cup \mathcal{E}_{-}^{1} \cup \mathcal{E}_{+}^{2} \cup \mathcal{E}_{-}^{2} \\
\mathbf{n} \times \nabla u_{1} & =0 & \text { on } \partial \Omega \backslash\left(\mathcal{E}_{+}^{1} \cup \mathcal{E}_{-}^{1} \cup \mathcal{E}_{+}^{2} \cup \mathcal{E}_{-}^{2}\right)
\end{array}\right.
$$

In the above model, skin-electrode contact impedance [61] is ignored for simplicity.

The principle of reciprocity invented by Helmholtz [28] is a very useful tool in bioimpedance. The reciprocity principle says that the voltage difference of $u_{1}$ between the pair of inner electrodes is the same as the voltage difference of $u_{2}$ between the pair of outer electrodes, where $u_{2}$ is a solution of (23) with interchanging subindex 1 and 2 .

Theorem 1 Let $u_{1}$ be a solution of (23) and $u_{2}$ be a solution of (23) with interchanging subindex 1 and 2. Then, we have the following reciprocity principle:

$$
\left.u_{1}\right|_{\mathcal{E}_{+}^{2}}-\left.u_{1}\right|_{\mathcal{E}_{-}^{2}}=\left.u_{2}\right|_{\mathcal{E}_{+}^{1}}-\left.u_{2}\right|_{\mathcal{E}_{-}^{1}}
$$

Proof For simplicity, we assume that all electrodes are the same size. The reciprocity principle can be shown by the following integration by parts:

$$
\begin{aligned}
\left.u_{1}\right|_{\mathcal{E}_{+}^{2}}-\left.u_{1}\right|_{\mathcal{E}_{-}^{2}} & =\frac{1}{\left|\mathcal{E}_{+}^{1}\right|}\left(\int_{\mathcal{E}_{+}^{2}} u_{1} d s-\int_{\mathcal{E}_{-}^{2}} u_{1} d s\right) \\
& =\frac{1}{\left|\mathcal{E}_{+}^{1}\right| I} \int_{\partial \Omega} u_{1} \mathbf{n} \cdot A \nabla u_{2} d s \\
& =\frac{1}{\left|\mathcal{E}_{+}^{1}\right| I} \int_{\Omega} \nabla u_{1} \cdot A \nabla u_{2} d \mathbf{x} \\
& =\frac{1}{\left|\mathcal{E}_{+}^{1}\right| I} \int_{\partial \Omega} u_{2} \mathbf{n} \cdot A \nabla u_{1} d s \\
& =\frac{1}{\left|\mathcal{E}_{+}^{1}\right|}\left(\int_{\mathcal{E}_{+}^{1}} u_{2} d s-\int_{\mathcal{E}_{-}^{1}} u_{2} d s\right) \\
& =\left.u_{2}\right|_{\mathcal{E}_{+}^{1}}-\left.u_{2}\right|_{\mathcal{E}_{-}^{1}} .
\end{aligned}
$$

\subsection{Electric impedance myography (EIM)}

Electrical impedance myography (EIM) is a technique for the assessment of muscle admittivity $A$ in order to evaluating neuromuscular diseases both for their diagnosis and for their ongoing assessment of the progress or therapeutic intervention $[49,52,54]$. Since muscle cells are composed of muscle fibers, 
they have anisotropic properties in which admittivity $A$ varies depending on the direction of the muscle fibers $[16,17]$. So muscle admittivity $A$ has two aspects; along and across the muscle fiber. When the electric field is formed along the muscle fiber direction, the admittivity of the muscle that affect the formation of the electromagnetic field is called longitudinal admittivity and denoted by $A_{\text {longi }}:=\sigma_{\text {longi }}+i \omega \epsilon_{0} \epsilon_{\text {longi }}$ with vacuum permittivity $\epsilon_{0}=8.85 \times 10^{-12} \mathrm{~F} \cdot \mathrm{m}^{-1}$, longitudinal conductivity $\sigma_{\text {longi }}$ and relative permittivity $\epsilon_{\text {longi }}$. Similarly, we call transverse admittvity $A_{\text {trans }}:=\sigma_{\text {trans }}+i \omega \epsilon_{0} \epsilon_{\text {trans }}$, conductivity $\sigma_{\text {trans }}$, and relative permittivity $\epsilon_{\text {trans }}$, in the case when electric field is formed across the muscle fiber. Thereby, the admittivity of muscle is anisotropy and it can be written as matrix, for example when muscle fiber lies along $x_{1}$-axis, then $a_{11}=A_{\text {longi }}, a_{22}=A_{\text {trans }}$, $a_{33}=A_{\text {trans }}$, and remaining $a_{i j}=0$ (Fig. 6). According to the [22] (in vivo study of rat muscle), longitudinal conductivity is 10 times higher than that of transverse, i.e. $\sigma_{\text {longi }} \approx 10 \sigma_{\text {trans }}$.

Note that the coefficient $A$ is complex anisotropy. So, the real and imaginary parts of $u$ [solution of (23)] gives system of equations. If anisotropic ratios of real and imaginary parts of $A$ are same, then the system of equations can be separated.

Observation 3.1 Let $u$ be a solution of (7) and for simplicity muscle fiber assume to be on $x_{1}$-axis. Define the anisotropy ratio of real and imaginary parts of $A$ as $\alpha^{2}:=\sigma_{\text {trans }} / \sigma_{\text {longi }}$ and $\beta^{2}:=\epsilon_{\text {trans }} / \epsilon_{\text {longi }}$, respectively. In general from the governing equation $\nabla \cdot((\sigma+i \lambda) \nabla(v+i w))=0$, we have

$$
\nabla \cdot(\sigma \nabla v-\lambda \nabla w)+i \nabla \cdot(\lambda \nabla v-\sigma \nabla w)=0,
$$

where $\sigma=\mathfrak{R}(A), \lambda=\mathfrak{I}(A)$, and $u=v+i w$. Then, we have a system of equations as below

$$
\left\{\begin{array}{l}
\left(\left(1 / \alpha^{2}\right) \partial_{1}^{2} v+\partial_{2}^{2} v+\partial_{3}^{2} v\right)-\varpi\left(\left(1 / \beta^{2}\right) \partial_{1}^{2} w+\partial_{2}^{2} w+\partial_{3}^{2} w\right)=0 \\
\varpi\left(\left(1 / \beta^{2}\right) \partial_{1}^{2} v+\partial_{2}^{2} v+\partial_{3}^{2} v\right)+\left(\left(1 / \alpha^{2}\right) \partial_{1}^{2} w+\partial_{2}^{2} w+\partial_{3}^{2} w\right)=0
\end{array}\right.
$$

where $\varpi=\epsilon_{\text {trans }} / \sigma_{\text {trans. If }} \alpha^{2}=\beta^{2}$, the above system of equations can be separated as
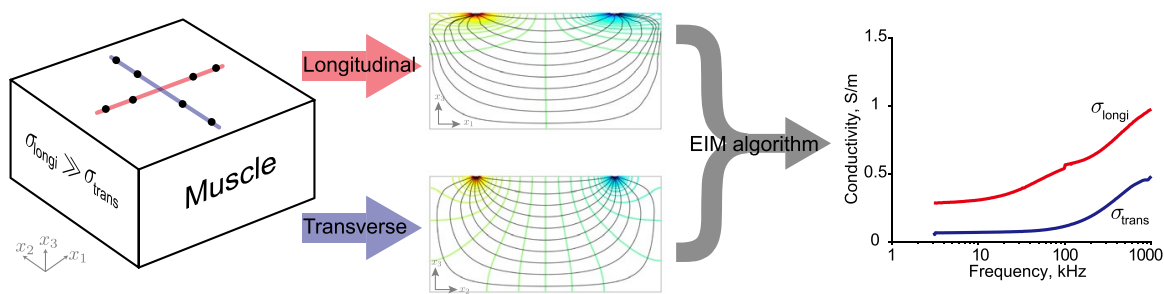

Fig. 6 Simple EIM model with a prior knowledge of muscle fiber direction in $\mathbb{R}_{-}^{3}$ with direct contact on the muscle. Electrodes are aligned on longitudinal direction (small black circles on a red line in left image) and transverse direction (small black circles on a blue line in left image). The right most plot is estimated conductivity of ovine gluteus medius from [38] 


$$
\left\{\begin{array}{c}
\left(1 / \alpha^{2}\right) \partial_{1}^{2} v+\partial_{2}^{2} v+\partial_{3}^{2} v=0 \\
\left(1 / \alpha^{2}\right) \partial_{1}^{2} w+\partial_{2}^{2} w+\partial_{3}^{2} w=0
\end{array}\right.
$$

In order to estimate $A$, EIM uses set of voltage differences with dividing current amplitude:

$$
Z:=\left(\left.u_{1}\right|_{\mathcal{E}_{+}^{2}}-\left.u_{1}\right|_{\mathcal{E}_{-}^{2}}\right) / I
$$

which is called impedance with same notations in the Theorem 1. Due to the anisotropic property of $A$, it is required to have at least two impedances, for example impedance measured on longitudinal and transverse directions. The measured impedance is a some sense of average of both longitudinal and transverse admittivity.

Observation 3.2 For the ease of explanation, we consider the half space domain $\Omega=\mathbb{R}_{-}^{3}$ (or free space $\mathbb{R}^{3}$ ), same anisotropy ratio $\alpha^{2}=\beta^{2}$, and four linearly aligned electrodes with spacing $a, b$, and $a$. When the electrodes alignment angle is $\xi$ and muscle fiber direction is $\theta$, the measured impedance $Z$ can be represented by [53]

$$
Z=\frac{1}{F_{\text {geom }} \sqrt{\cos ^{2}(\theta-\xi)+\alpha^{2} \sin ^{2}(\theta-\xi)} \sqrt{A_{\text {longi }} A_{\text {trans }}}}
$$

where the geometry factor $F_{\text {geom }}=F_{\text {domain }} / F_{\text {eled }}$, the domain factor $F_{\text {domain }}=2 \pi$ (or $F_{\text {domain }}=4 \pi$ for $\left.\Omega=\mathbb{R}^{3}\right)$, and the electrode factor $F_{\text {eled }}:=\frac{2 b}{a(a+b)}$.

Hence the inverse problem of EIM is to estimate $A_{\text {trans }}$ and $A_{\text {longi }}$ from a set of measured impedance of several measuring angle $\theta[38,39]$.

\subsection{Electrical impedance tomography (EIT)}

Electrical impedance tomography (EIT) aims to visualize the distribution of (isotropic) coefficient $A$ with meaning that $A$ is human body using finite number of surface electrodes. One of most successful clinical application in EIT is lung ventilation monitoring, i.e. $A$ is lung (Fig. 7). For the reconstruction of the distribution of $A$, a set of Neumann-to-Dirichlet map is constructed with several choices of current injection electrodes and corresponding voltage measuring electrodes for each pair of current injection electrodes. For the sake of simple explanation, let $\left\{\mathcal{E}_{1}, \mathcal{E}_{2}, \ldots, \mathcal{E}_{16}\right\}$ be a set of surface electrodes attached on the boundary of human body $\Omega$ with adjacent pairs of current and voltage electrodes. Then, we have the following Neumann-to-Dirichlet data:

$$
\mathfrak{L}:=\left\{Z^{1,3}, Z^{1,4}, \ldots, Z^{1,15}, Z^{2,4}, \ldots, \ldots, Z^{16,14}\right\}
$$

where $Z^{i, j}:=\left.u_{i}\right|_{\mathcal{E}_{j}}-\left.u_{i}\right|_{\mathcal{E}_{j+1}}$ and $u_{i}$ is the solution of 

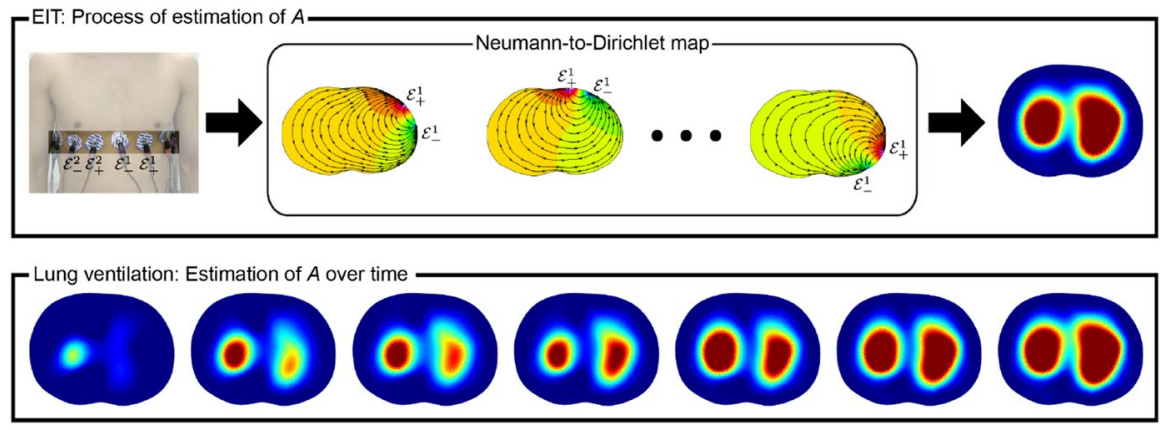

Fig. 7 EIT model for lung ventilation is to visualize distribution of time change of isotropic $A$. During lung ventilation, pulmonary conductivity $A$ changes due to conversion of air in the alveoli

$$
\left\{\begin{array}{rlrl}
\nabla \cdot\left(A \nabla u_{i}\right) & =0 & \text { in } \Omega \\
\mathbf{n} \cdot\left(A \nabla u_{i}\right) & =0 & \text { on } \partial \Omega \backslash \cup_{k=1}^{16} \mathcal{E}_{k} \\
\int_{\mathcal{E}_{k}} \mathbf{n} \cdot\left(A \nabla u_{i}\right) d s & =0 & \text { for } k \in\{1, \ldots, 16\} \backslash\{i, i+1\} \\
\left.\left(u_{i}+z_{i, k} \mathbf{n} \cdot\left(A \nabla u_{i}\right)\right)\right|_{\mathcal{E}_{k}} & =U_{i, k} & \text { on } \mathcal{E}_{\ell} \text { for } \ell=1, \ldots, 16 \\
\int_{\mathcal{E}_{i}} \mathbf{n} \cdot\left(A \nabla u_{i}\right) d s & =I=-\int_{\mathcal{E}_{i+1}} \mathbf{n} \cdot\left(A \nabla u_{i}\right) d s
\end{array}\right.
$$

where $z_{i, k}$ is skin-electrode contact impedance [61]. Hence, the inverse problem of EIT is to reconstruct (equivalent isotropic admittivity) $A$ from Neumann-to-Dirichlet data $\mathfrak{Q}$ in (26). There are several EIT algorithm as in [1, 5, 27, 30, 32, 42].

\section{Discussions and conclusions}

In medical imaging, mathematical methodologies have evolved to improve our ability to visualize various physical phenomena and features accurately and reliably. The analysis of PDE models often plays an important role in the achievement of major advances in these areas. Technical advances have been followed by theoretical progress aimed at understanding the solution's structure. However, the subject of partial differential equations should not be studied in isolation, because much intuition comes from a thorough understanding of applications.

Let us briefly discuss the inverse problem of reconstructing the equivalent isotropic conductivity of $A$. In spite of novel theoretical results guaranteeing a unique identification of $A$ from the Neumann-to-Dirichlet data [8, 45, 62], numerous experiences have shown that static EIT for recovering isotropic admittivity $A$ has fundamental drawbacks because EIT data depends strongly on the boundary geometry and electrode positions, whereas it is much less sensitive to a local perturbation of isotropic $A$ away from the measuring electrodes [56]. In 2003, Magnetic Resonance Electrical Impedance Tomography (MREIT) was invented to deal with the wellknown ill-posedness of the image reconstruction problem of EIT [55]. Mathematicsoriented research overcomes technical barriers in electrical tissue property imaging. This paper observed that $\nabla \ln A \cdot(A \nabla u \times(0,0,1))$ with $u$ being a solution of (7) is 
measurable quantity using MRI system so that it can probe changes in the logarithm of the isotropic $A$ along any equipotential curve in each imaging slice. Here, $(0,0,1)$ is the direction of the main magnetic field of MRI scanner. This method uses two different Neumann data $g_{1}, g_{2}$ so that the area of the parallelogram made by these two vector fields $\nabla u_{1}$ and $\nabla u_{2}$ is non-zero at every position in the imaging slice. Taking advantage of these mathematical observations, they found a representation formula for the equivalent isotropic $A$ which can offer state-of-the-art conductivity imaging using MRI animal and human experiments [56, 60, 63].

There have been developed numerous bioimpedance-based health care systems such as body fat assessment [31], stroke volume monitoring, hypoventilation monitoring [7], lung monitoring [15], and so on. All of these are tied to the effective property of $A$, that is the coefficient of the elliptic PDE. Recently, bioimpedance techniques are used for non-destructive continuous monitoring of in vitro chondrogenesis for the production of high-performance engineered cartilage of clinical use and its quality control [41, 47].

Impedance imaging techniques have been used various areas beside the biomedical area such as ground monitoring [13, 40, 48] and industrial process monitoring [6]. Chipot et al [12] developed a pressure-sensitive conductive fabric sensor, which is based on a design of a composite fabric consisting of an electrically conductive yarn and a sponge-like non-conductive fabric with high pore density. In this model, the conductive yarn is woven in a wavy pattern to possess a pressure-sensitive conductive property (being capable of changing its effective electrical property of the admittivity $A$ due to an applied pressure, in the sense of homogenization theory).

Developing mathematical models with practical significance and value requires the fusing of the knowledge and techniques of traditional engineering fields with pure and applied mathematics [57]. It is necessary to understand practical limitations imposed by measurement methods in mathematical models.

Acknowledgements A.J. was supported by NRF Grant 2017R1E1A1A03070653. J.K.S. was supported by the National Research Foundation of Korea (NRF) Grants 2015R1A5A1009350 and 2017R1A2B20005661.

\section{Compliance with ethical standards}

Conflict of interest The author has no conflict of interest to declare.

Ethical approval This article does not contain any studies with human participants or animals performed by any of the authors.

Open Access This article is licensed under a Creative Commons Attribution 4.0 International License, which permits use, sharing, adaptation, distribution and reproduction in any medium or format, as long as you give appropriate credit to the original author(s) and the source, provide a link to the Creative Commons licence, and indicate if changes were made. The images or other third party material in this article are included in the article's Creative Commons licence, unless indicated otherwise in a credit line to the material. If material is not included in the article's Creative Commons licence and your intended use is not permitted by statutory regulation or exceeds the permitted use, you will need to obtain permission directly from the copyright holder. To view a copy of this licence, visit http://creativecommons.org/licen ses/by/4.0/. 


\section{References}

1. Adler, A., Arnold, J.H., Bayford, R., Borsic, A., Brown, B., Dixon, P., Faes, T.J., Frerichs, I., Gagnon, H., Gärber, Y., Grychtol, B.: GREIT: a unified approach to 2D linear EIT reconstruction of lung images. Physiol. Meas. 30(6), S35 (2009)

2. Allaire, G.: Homogenization and two-scale convergence. SIAM J. Math. Anal. 23, 1482-1518 (1992)

3. Ammari, H., Garnier, J., Giovangigli, L., Jing, W., Seo, J.K.: Spectroscopic imaging of a dilute cell suspension. J. de Mathématiques Pures et Appliquées. 105(5), 603-661 (2016)

4. Astala, K., Päivärinta, L.: Calderón's inverse conductivity problem in the plane. Ann. Math. 163(1), 265-299 (2006)

5. Barber, D.C., Brown, B.H., Avis, N.J.: Image reconstruction in electrical impedance tomography using filtered back-projection. In: 1992 14th Annual International Conference of the IEEE Engineering in Medicine and Biology Society, vol. 5, pp. 1691-1692 (1992)

6. Beck, M.S.: Process tomography: principles, techniques and applications. Butterworth-Heinemann, Oxford (2012)

7. Bilab web. https://bilabhealthcare.com. Accessed 3 Feb 2020

8. Calderón, A.P.: On an inverse boundary value problem. In: Seminar on numerical analysis and its applications to continuum physics. Soc. Brasileira de Matemàtica. 65-73 (1980)

9. Carstensen, E.L., Cox, H.A., Mercer, W.B., Natale, L.A.: Passive electrical properties of microorganisms: I. Conductivity of Escherichia coli and Micrococcus lysodeikticus. Biophys. J. 5(3), 289300 (1965)

10. Chin, A.B., Garmirian, L.P., Nie, R., Rutkove, S.B.: Optimizing measurement of the electrical anisotropy of muscle. Muscle Nerve 37(5), 560-565 (2008)

11. Chipot, M.: Elliptic equations: an introductory course. Springer, Berlin (2009)

12. Chipot, M., Lee, K., Seo, J.K.: Mathematical model of conductive fabric-based flexible pressure sensor. Appl. Math. Model. 48, 775-786 (2017)

13. Daily, W., Ramirez, A., LaBrecque, D., Nitao, J.: Electrical resistivity tomography of vadose water movement. Water Resour. Res. 28(5), 1429-1442 (1992)

14. Davit, Y., Bell, C.G., Byrne, H.M., Chapman, L.A., Kimpton, L.S., Lang, G.E., Leonard, K.H., Oliver, J.M., Pearson, N.C., Shipley, R.J., Waters, S.L.: Homogenization via formal multiscale asymptotics and volume averaging: How do the two techniques compare? Adv. Water Resour. 62, 178-206 (2013)

15. Draeger web. https://www.draeger.com/en_aunz/Hospital/EIT-Lung-Monitoring. Accessed 3 Feb 2020

16. Epstein, B.R., Foster, K.R.: Anisotropy in the dielectric properties of skeletal muscle. Med. Biol. Eng. Comput. 21(1), 51 (1983)

17. Fatt, P.: An analysis of the transverse electrical impedance of striated muscle. Proc. R. Soc. Lond. B Biol. Sci. 159(977), 606-651 (1964)

18. Fricke, H.: A mathematical treatment of the electric conductivity and capacity of disperse systems I. The electric conductivity of a suspension of homogeneous spheroids. Phys. Rev. 24(5), 575-587 (1924)

19. Fricke, H.: A mathematical treatment of the electric conductivity and capacity of disperse systems II. The capacity of a suspension of conducting spheroids surrounded by a non-conducting membrane for a current of low frequency. Phys. Rev. 26(5), 678-681 (1925)

20. Fricke, H.: The Maxwell-Wagner dispersion in a suspension of ellipsoids. J. Phys. Chem. 57(9), 934-937 (1953)

21. Geddes, L.A., Baker, L.E.: The specific resistance of biological material: a compendium of data for the biomedical engineer and physiologist. Med. Biol. Eng. 5, 271-93 (1967)

22. Gielen, F.L.H., Wallinga-de Jonge, W., Boon, K.L.: Electrical conductivity of skeletal muscle tissue: experimental results from different muscles in vivo. Med. Biol. Eng. Comput. 22(6), 569-577 (1984)

23. Gladwell, G.M.L., Coen, S.: A Chebyshev approximation method for microstrip problems. IEEE Trans. Microw. Theory Tech. 23(11), 865-870 (1975)

24. Grimnes, S., Martinsen, O.G.: Bioimpedance and bioelectricity basics. Elsevier, Oxford (2008)

25. Grimnes, S., Martinsen, Ø.G.: Bioimpedance. In: Wiley Encyclopedia of Biomedical Engineering (2006) 
26. Hadamard, J.: Sur le principe de Dirichlet. Bull. Soc. Math. France 34, 135-138 (1906)

27. Harrach, B.: Recent progress on the factorization method for electrical impedance tomography. Computational and mathematical methods in medicine 2013. Article ID 425184 (2013)

28. Helmholtz, H.: Handbuch der physiologischen Optik, first edition cited by Planck, vol. 1. Leopold Voss, Leipzig, p 169 (1856)

29. Holder, D.: Electrical impedance tomography: methods, history and applications. CRC Press, Boca Raton (2004)

30. Hua, P., Webster, J.G., Tompkins, W.J.: A regularised electrical impedance tomography reconstruction algorithm. Clin. Phys. Physiol. Meas. 9(4A), 137 (1988)

31. Inbody technology. http://www.inbody.com/global/main/Main.aspx. Accessed 3 Feb 2020

32. Isaacson, D., Mueller, J.L., Newell, J.C., Siltanen, S.: Reconstructions of chest phantoms by the D-bar method for electrical impedance tomography. IEEE Trans. Med. Imaging 23(7), 821-828 (2004)

33. Kenig, C., Sjostrand, J., Uhlmann, G.: The Calderon problem with partial data. Ann. Math. 165, 567-591 (2007)

34. Kim, S., Lee, E.J., Woo, E.J., Seo, J.K.: Asymptotic analysis of the membrane structure to sensitivity of frequency-difference electrical impedance tomography. Inverse Prob. 28(7), 075004 (2012)

35. Kohn, R., Vogelius, M.: Determining conductivity by boundary measurements. Commun. Pure Appl. Math. 37(3), 289-298 (1984)

36. Kyle, U.G., Bosaeus, I., De Lorenzo, A.D., Deurenberg, P., Elia, M., Gómez, J.M., Heitmann, B.L., Kent-Smith, L., Melchior, J.C., Pirlich, M., Scharfetter, H.: Bioelectrical impedance analysis-part I: review of principles and methods. Clin. Nutr. 23(5), 1226-1243 (2004)

37. Kyle, U.G., Bosaeus, I., De Lorenzo, A.D., Deurenberg, P., Elia, M., Gómez, J.M., Heitmann, B.L., Kent-Smith, L., Melchior, J.C., Pirlich, M., Scharfetter, H.: Bioelectrical impedance analysis-part II: utilization in clinical practice. Clin. Nutr. 23(6), 1430-1453 (2004)

38. Kwon, H., Nagy, J.A., Taylor, R., Rutkove, S.B., Sanchez, B.: New electrical impedance methods for the in situ measurement of the complex permittivity of anisotropic biological tissues. Phys. Med. Biol. 62(22), 8616 (2017)

39. Kwon, H., Guasch, M., Nagy, J.A., Rutkove, S.B., Sanchez, B.: New electrical impedance methods for the in situ measurement of the complex permittivity of anisotropic skeletal muscle using multipolar needles. Sci. Rep. 9(1), 1-16 (2019)

40. Langer, R.E.: An inverse problem in differential equations. Bull. Am. Math. Soc. 39(10), 814-820 (1933)

41. Lee, E.J., Wi, H., McEwan, A.L., Farooq, A., Sohal, H., Woo, E.J., Seo, J.K., Oh, T.I.: Design of a microscopic electrical impedance tomography system for 3D continuous non-destructive monitoring of tissue culture. Biomed. Eng. Online. 13(1), 142 (2014)

42. Lee, K., Woo, E.J., Seo, J.K.: A fidelity-embedded regularization method for robust electrical impedance tomography. IEEE Trans. Med. Imaging 37(9), 1970-1977 (2017)

43. Maxwell, J.: A treatise on electricity and magnetism. MacMillan \& Co., Oxford at the Clarendon Press, London (1873)

44. Milton, G.W.: The theory of composites. Cambridge University Press, Cambridge (2002)

45. Nachman, A.I.: Reconstructions from boundary measurements. Ann. Math. 128(3), 531-576 (1988)

46. Nachman, A.I.: Global uniqueness for a two-dimensional inverse boundary value problem. Ann. Math. 143(1), 71-96 (1996)

47. Oh, T.I., Kim, C., Karki, B., Son, Y., Lee, E., Woo, E.J.: Non-destructive label-free continuous monitoring of in vitro chondrogenesis via electrical conductivity and its anisotropy. Biotechnol. Bioeng. 112(2), 422-427 (2015)

48. Ramirez, A., Daily, W., LaBrecque, D., Owen, E., Chesnut, D.: Monitoring an underground steam injection process using electrical resistance tomography. Water Resour. Res. 29(1), $73-87$ (1993)

49. Rehman, A.A., Elmore, K.B., Mattei, T.A.: The effects of alternating electric fields in glioblastoma: current evidence on therapeutic mechanisms and clinical outcomes. Neurosurg. Focus 38(3), E14 (2015)

50. Rellich, F.: Darstellung der Eigenwerte von $u+\lambda u=0$ durch ein Randintegral. Math. Z. 46, 635636 (1940)

51. Rothwell, E.J., Cloud, M.J.: Electromagnetics, 3rd edn. Taylor \& Francis CRC Press, Boca Raton (2008). (ISBN 978-1-4987-9656-9)

52. Rubinsky, B.: Irreversible electroporation in medicine. Technol. Cancer Res. Treat. 6(4), 255-259 (2007) 
53. Rush, S.: Methods of measuring the resistivities of anisotropic conducting media in situ. J. Res. Nat. Bureau Stand. C. Eng. Instrument C. 66, 217-222 (1962)

54. Rutkove, S.B.: Electrical impedance myography: background, current state, and future directions. Muscle Nerve 40(6), 936-946 (2009)

55. Seo, J.K., Yoon, J.R., Woo, E.J., Kwon, O.: Reconstruction of conductivity and current density images using only one component of magnetic field measurements. IEEE Trans. Biomed. Eng. 50(9), 1121-1124 (2003)

56. Seo, J.K., Woo, E.J.: Magnetic resonance electrical impedance tomography (MREIT). SIAM Rev. 53(1), 40-68 (2011)

57. Seo, J.K., Woo, E.J.: Nonlinear inverse problems in imaging. Wiley, Hoboken (2013)

58. Seo, J.K., Woo, E.J., Katcher, U., Wang, Y.: Electro-magnetic tissue properties MRI. Imperial College Press, London (2013)

59. Seo, J.K., Bera, T.K., Kwon, H., Sadleir, R.: Effective admittivity of biological tissues as a coefficient of elliptic PDE. Comput. Math. Methods Med. 2013, 353849 (2013)

60. Seo, J.K., Woo, E.J.: Electrical tissue property imaging at low frequency using MREIT. IEEE Trans. Biomed. Eng. 61(5), 1390-1399 (2014)

61. Spach, M.S., Barr, R.C., Havstad, J.W., Long, E.C.: Skin-electrode impedance and its effect on recording cardiac potentials. Circulation 34(4), 649-656 (1966)

62. Sylvester, J., Uhlmann, G.: A global uniqueness theorem for an inverse boundary value problem. Ann. Math. 125, 153-169 (1987)

63. Woo, E.J., Seo, J.K.: Magnetic resonance electrical impedance tomography (MREIT) for high-resolution conductivity imaging. Physiol. Meas. 29(10), R1-R26 (2008)

Publisher's Note Springer Nature remains neutral with regard to jurisdictional claims in published maps and institutional affiliations. 